\title{
Soy isoflavone: The multipurpose phytochemical (Review)
}

\author{
QINGLU WANG ${ }^{1}$, XIAOYUE GE $^{1}$, XUEWEN TIAN $^{2}$, YUJUN ZHANG $^{1}$, JIE ZHANG $^{1}$ and PINGPING ZHANG ${ }^{1}$ \\ ${ }^{1}$ Key Laboratory of Biomedical Engineering and Technology of Shandong High School, Shandong Wanjie Medical College, \\ Zibo, Shandong 255213; ${ }^{2}$ Shandong Research Center of Sports Science, Jinan, Shandong 250102, P.R. China
}

Received April 10, 2013; Accepted May 21, 2013

DOI: $10.3892 /$ br.2013.129

\begin{abstract}
Soy isoflavones are compounds found in soybean and soybean products. They have been reported to possess numerous physiological properties, such as antitumor, antimenopausal (female) osteoporosis and anti-aging. They have also been reported to improve learning and memory skills in menopausal women and aid in the prevention and treatment of heart disease, diabetes and Kawasaki disease (KD). In this review, the effects of soy isoflavones on various diseases were analyzed. Based on the analysis, it was hypothesized that the function of soybean isoflavones in the prevention and treatment of various diseases results from their phytoestrogen and antioxidant properties. However, due to their phytoestrogen properties, it is recommended that the risks of soy isoflavone intake as food and/or medical treatment be further evaluated.
\end{abstract}

\section{Contents}
1. Introduction
2. Effects of soy isoflavones on cancer
3. Osteoporosis and soy isoflavones
4. Effects of soy isoflavones on learning and memory
5. Effects of soy isoflavones on coronary heart disease
6. Effects of soy isoflavones on diabetes
7. Anti-photoaging effects of soy isoflavones
8. Kawasaki disease and soy isoflavones
9. Risks of soy isoflavones
10. Conclusions

\section{Introduction}

Asian populations have consumed foods made from soy beans for centuries, whereas in the West, certain subpopulations,

Correspondence to: Dr Qinglu Wang, Key Laboratory of Biomedical Engineering and Technology of Shandong High School, Shandong Wanjie Medical College, Boshan Economic Development Zone, Zibo, Shandong 255213, P.R. China

E-mail: wqlzcq@gmail.com

Key words: soy isoflavone, phytochemical, disease, phytoestrogen, antioxidant ability specifically Seventh-day Adventists and vegetarians, have used soyfoods for $\sim 100$ years, although the quintessential soyfood tofu was first introduced on a large scale to the general US population in the early 1970s. Health-conscious and ecologically-minded consumers were particularly attracted to soy at that time since it was perceived as a source of high quality protein, low in saturated fat, that was more efficiently produced compared to animal sources of protein. A significant increase in soyfood consumption occurred during the last decade of the 20th century, mainly due to the belief among many consumers that soyfoods may offer health benefits, independent of their nutrient content. This increased interest is best viewed in the context of the general recognition that plants contain large quantities of potentially beneficial, non-nutritive, biologically active components, commonly referred to as phytochemicals. This knowledge led to the concept of functional foods [initially referred to as designer foods by the National Cancer Institute (NCI)] and to soy being one of the first foods widely acknowledged to fall into this category (1). Soy isoflavones contain 12 different isoforms that are divided into four chemical forms: aglycone (daidzein, genistein and glycitein), glucoside (daidzin, genistin and glycitin), acetylglucoside (acetyldaidzin, acetylgenistin and acetylglycitin) and malonylglucoside (malonyldaidzin, malonylgenistin and malonylglycitin). Soy isoflavones were demonstrated to possess numerous biological functions, such as antioxidant (2), inhibitory on cancer cell proliferation (3), anti-inflammatory (4) and preventive of coronary heart disease (5) and osteoporosis (6).

Over the last 22 years, there has been a considerable amount of research on the health effects of soy consumption, which may be largely attributed to the presence of isoflavones in the soybean. Isoflavones first came to the attention of the scientific community in the 1940 s, as a result of fertility problems observed in sheep grazing on a type of isoflavone-rich clover (1). In the 1950s, due to their estrogenic effects in rodents, isoflavones were investigated for use as possible growth promoters by the animal feed industry, although shortly thereafter it was demonstrated that isoflavones may also act as antiestrogens (1). Despite this early study, it was not until the 1990s, mainly due to research sponsored by the US NCI, that the role of soyfoods in disease prevention started to attract significant attention. Subsequently, isoflavones and soyfoods were investigated on their ability to alleviate hot flashes and inhibit bone loss in postmenopausal women. In 1995, soy protein attracted worldwide attention for its ability to lower cholesterol levels (1). At that same time, isoflavones started to be widely discussed as 
potential alternatives to conventional hormone replacement therapy (1). In 2002, it was hypothesized that individuals harbouring intestinal bacteria capable of converting the soybean isoflavone daidzein into the isoflavan equol were more likely to benefit from soy intake (1). More recently, in vitro and animal studies have raised questions regarding the safety of exposure to isoflavones for certain subsets of the population, although human data are largely inconsistent with these concerns (1).

\section{Effects of soy isoflavones on cancer}

Melanoma and breast tumor cells are capable of degrading the extracellular matrix via a proteolytic cascade that includes urokinase-type plasminogen activator (uPA) and matrix metalloproteinases (MMPs). At non-cytotoxic concentrations (0.1-50 $\mu \mathrm{M})$, genistein dose-dependently promoted spindle-cell morphology and significantly reduced motility in the two cell lines. Genistein inhibited uPA secreted by F3II cell monolayers, while inducing an increase in the proteolytic activity of B16 cells. By contrast, the compound did not modify the MMP-9 and -2 secretion by tumor cells. These data suggest that tumor cell migration and proteolysis are associated with the antitumor and antiangiogenic activity of the soy isoflavone genistein (7).

Recent studies have demonstrated that soy and isoflavone intake exerts a protective effect against postmenopausal breast cancer (8-10). The overexpression of HER 2 promotes the malignant transformation of breast epithelial cells. Genistein enhanced the cytotoxic effect of adriamycin (ADR) at low doses (less than $\mathrm{IC}_{50}$ ) against the human breast cancer cell. This enhancing effect was mainly attributed to the increase of necrotic-like, rather than apoptotic, cell death. In conjugation with this event, significant inactivation of HER2 and Akt in the breast cancer cell was caused by the combination of genistein and ADR. These results suggest that genistein enhances the necrotic-like cell death of breast cancer cells through the inactivation of the HER2 receptor and Akt in combination with ADR (11). Statistical data demonstrated that high isoflavone intake (combined $\mathrm{RR} / \mathrm{OR}=0.68,95 \% \mathrm{CI}$ : 0.52-0.89) may be associated with reduced breast cancer risk in Asian populations, particularly in postmenopausal women. However, results of studies conducted on Western populations indicated no significant differences, which may be due to the low intake of isoflavone (12).

A previous study by Akaza et al (13) was the first to confirm that there is an association between prostate cancer and isoflavone intake. In a later study, Kurahashi et al (14) demonstrated that isoflavone intake was associated with a decreased risk of localized prostate cancer. However, a study by Perabo et al (15) reported no convincing clinical proof or evidence that genistein may be beneficial in prostate cancer therapy. Between 2008 and 2012, five out of six studies reported a significant association of isoflavones with a decreased risk of prostate cancer (16), two of which consistently demonstrated that daidzein converted to equol by intestinal bacteria leads to a significantly reduced risk of prostate cancer (16).

\section{Osteoporosis and soy isoflavones}

The first clinical trial in this area to report a benefit was published in 2001 (17). The prevalence of age-related bone loss is higher in women compared to men and in $25-30 \%$ of aging women this loss results in major orthopedic problems (18). Natural or surgical menopause results in an initial phase of rapid bone loss followed by a period of slower skeletal deterioration (19). This rapid phase of bone loss occurs within the first 10 years following the cessation of menses or surgical removal of the ovaries (20). The ovarian hormone deficiency associated with menopause results in an increased rate of bone turnover and leads to an imbalance between resorption and formation, thereby accelerating bone loss (21). A previous meta-analysis reported that soy isoflavones significantly increased the bone mineral density by $54 \%$ and decreased the bone resorption marker urinary deoxypyridinoline by $23 \%$ compared to baseline in women. A sensitivity analysis indicated that the effect of soy isoflavones on bone mineral density and deoxypyridinoline was significant. Postmenopausal women experience a sharp decrease in estrogen concentration, leading to an increased rate of bone remodeling, which is associated with decreased bone mineral density and increased risk of fractures $(20,22,23)$.

\section{Effects of soy isoflavones on learning and memory}

A previous study indicated that estrogen use increases the performance on certain tests of cognition, particularly in postmenopausal women (20). Short-term high-dose soy bean intake altered the levels of total plasma testosterone and improved spatial cognition in women (24). Isoflavone supplementation exerted a favorable effect on cognitive function, particularly verbal memory, in postmenopausal women (25). Consumption of dietary phytoestrogens resulting in high plasma isoflavone levels may significantly affect sexually dimorphic brain regions, anxiety, learning and memory (26). Dietary soy-derived phytoestrogens may affect learning and memory and alter the expression of proteins involved in neural protection and inflammation in rats (27). Soy isoflavones may also affect the cholinergic system of the brain and reduce age-related neuronal loss and cognition decline in male rats (28). There are proposed mechanisms for the neuroprotective effects of isoflavones. Genistein protects cells from $\mathrm{H}_{2} \mathrm{O}_{2}$-induced toxicity (29). $\mathrm{H}_{2} \mathrm{O}_{2}$ is a reactive oxygen species (ROS) that may cause neuron damage (30-33). Genistein, a phytoestrogen capable of crossing the blood-brain barrier, has been reported to exert an antioxidant effect against the insults of ultraviolet (UV) radiation and chemicals (34). This antioxidant effect of soy may protect against neurodegenerative diseases. Previous findings suggested that the mechanisms by which phytoestrogens, particularly genistein, protect neuronal cells include its antioxidant activity, as well as the activation of estrogen receptors (ERs) and upregulation of brain-derived neurotrophic factors (34). This improvement in cognitive ability in phytoestrogen-treated females may be due in part to the increased presence of choline acetyltransferase mRNA in the frontal cortex, which was associated with protection and enhancement of cognitive function (35). Furthermore, phytoestrogens significantly affect the brain calcium-binding protein calbindin (CALB), which acts as a buffer by binding intracellular calcium and plays an important role in mediating cell proliferation, programmed cell death (apoptosis) and neurotoxicity $(27,36)$. Soy isoflavones were shown to improve learning and memory function in menopausal women and it 
was recently reported that they may improve learning and memory impairment induced by $A \beta 1-42$ in rats, maintain $A \beta$ homeostasis in the brain, regulate the disordered expression of RAGE/LRP-1, and restrain RAGE-related NF- $\kappa$ B and inflammatory cytokine activation in neurovascular structures $(27,36)$. These results suggested that soy isoflavones may protect $\mathrm{A} \beta$-impaired learning and memory in rats and the mechanism of action may be associated with the regulation of vascular $A \beta$ transportation and vascular inflammatory reaction.

\section{Effects of soy isoflavones on coronary heart disease}

Soy protein-containing foods are a rich source of isoflavone phytoestrogens, such as genistein and daidzein. There is growing interest in these substances, since a high dietary intake of soy-containing foods has been associated with lower rates of chronic diseases, including coronary heart disease (37). Soy phytoestrogens bind weakly to the estrogen receptor (ER) and some bind more strongly to ER- $\beta$ compared to ER- $\alpha$. A previous meta-analysis indicated that isoflavone phytoestrogens decreased plasma cholesterol concentrations in subjects with initially elevated levels, but had little effect on subjects with normal serum cholesterol concentrations (37). These substances may also exert beneficial effects on arterial endothelial function, which was also confirmed by Beavers et al (38). In addition to these potentially antiatherogenic effects, numerous laboratories are investigating other possible mechanisms, including the antioxidant and antiproliferative properties of these substances (39). A previous study by Tikkanen and Adlercreutz (37) demonstrated that dietary supplementation with soy-derived isoflavones reduced the in vitro oxidation susceptibility of low-density lipoprotein (LDL). It was hypothesized that lipophilic phytoestrogen derivatives may be incorporated into LDLs, increasing their oxidation resistance and antiproliferative efficacy ex vivo, which theoretically are antiatherogenic effects. Another study by Chan et al (40) demonstrated that a 12-week isoflavone treatment reduced the serum levels of high-sensitivity C-reactive protein and improved brachial flow-mediated dilatation in patients with clinically manifest atherosclerosis, thus reversing their endothelial dysfunction status. The authors of that study suggested that their findings may have important implications regarding the use of isoflavones as secondary prevention in patients with cardiovascular disease, in addition to conventional interventions.

\section{Effects of soy isoflavones on diabetes}

Type 2 diabetes is a result of chronic insulin resistance and loss of functional pancreatic $\beta$-cell mass. Over the past 10 years, numerous studies demonstrated that genistein possesses antidiabetic properties, such as direct effects on $\beta$-cell proliferation, glucose-stimulated insulin secretion and protection against apoptosis, independent of its functions as an estrogen receptor agonist, antioxidant, or tyrosine kinase inhibitor (41). Its effects are structure-specific and not common to all flavonoids (42). While there are limited data available on the effects of genistein consumption in diabetic humans, there are several animal and cell-culture studies that demonstrated a direct effect of genistein on $\beta$-cells at physiologically relevant concentrations $(<10 \mu \mathrm{M})(41)$. The effects appear to involve cAMP/PKA signaling and a previous study suggested an effect of gene expression on epigenetic regulation (41).

Genistein was shown to relieve diabetic peripheral painful neuropathy, revert proinflammatory cytokine and ROS overproduction and restore the nerve growth factor (NGF) content of the diabetic sciatic nerve. Genistein was also shown to restore the glutathione (GSH) content and the ratio of reduced to oxidized glutathione (GSH/GSSG), improve the antioxidant enzyme activities, decrease ROS and lipoperoxide levels in the brain and liver and restore the inducible and endothelial nitric oxide synthase (iNOS and eNOS, respectively) content and superoxide dismutase activity in the thoracic aorta. Hyperglycaemia and weight loss were not affected. Genistein was able to reverse diabetic allodynia, oxidative stress and inflammation, as well as ameliorate NGF content and vascular dysfunction, thus suggesting its possible therapeutic use for diabetes complications (43).

\section{Anti-photoaging effects of soy isoflavones}

A previous study demonstrated that the protective effects of soy isoflavones against UVB radiation may be related to their antioxidant activities (44). Moreover, the soy isoflavone extract inhibited UVB-induced keratinocyte death and suppressed UVB-induced intracellular $\mathrm{H}_{2} \mathrm{O}_{2}$ release, which reduced oxidative stress. Furthermore, it decreased epidermal thickness and inhibited COX-2 and proliferating cell nuclear antigen expression. The activation of p38, c-Jun N-terminal kinase and extracellular signal-regulated kinase 1/2 (ERK1/2) were triggered by UVB and counteracted by treatment with soy isoflavone extract (44-46).

\section{Kawasaki disease and soy isoflavones}

Kawasaki disease (KD) is a diffuse vasculitis occurring in children, with a predilection for the coronary arteries. Recent data from a genetic study emphasized the role of specific immune receptors in the pathogenesis of KD. The functions of the Fc $\gamma$ receptors $(\mathrm{Fc} \gamma \mathrm{Rs})$ are modulated by soy isoflavones, genistein in particular. Epidemiological data from Hawaiian populations suggested an association between soy consumption and KD. These observations form the basis of the hypothesis that isoflavones are involved in KD pathogenesis by modulating the function of the FcGRs and disrupting the balance between the activation and inhibition of the inflammatory response (47).

\section{Risks of soy isoflavones}

Despite the considerable enthusiasm regarding the potential health benefits of soyfoods and isoflavones, there is also growing concern regarding their safety, based largely on their estrogen-like properties. Isoflavones are classified as phytoestrogens and mixed estrogen agonists/antagonists as well as endocrine disruptors (48-50). Evaluations of isoflavone safety have been conducted by governmental and quasi-governmental agencies in several European countries, Japan and Israel and the European Food Safety Authority is currently conducting another evaluation (1). These concerns are based almost exclusively on in vitro and animal studies (human studies, including 
clinical and epidemiological data, are supportive of safety) and the most notable among these is that isoflavone-containing products pose a risk to estrogen-sensitive breast cancer patients and women at high-risk of developing this disease and that isoflavone exposure via the consumption of soy-based infant formula may negatively affect the long-term development of infants (51-52).

\section{Conclusions}

In this study, the properties and role of soybean isoflavones in the prevention and treatment of several diseases were presented, which comprise antitumor, antimenopausal (female) osteoporosis and antiaging properties, improvement of learning and memory skills of menopausal women, prevention and treatment of heart disease, diabetes and KD. Based on the assessment of these diseases under the effect of isoflavones, it was concluded that the function of soybean isoflavones in the prevention and treatment of various diseases largely results from their phytoestrogen and antioxidant properties. Although soybean isoflavones have been demonstrated to exert beneficial effects on the prevention and treatment of several diseases, possible adverse reactions have recently become the focus of attention. Therefore, more clinical trials are required to evaluate the effects of isoflavones on the prevention of disease development and their role in the treatment of various diseases, either alone or in combination with conventional therapeutic methods.

\section{Acknowledgements}

This study was supported by grants from the Program of Science and Technology Development of Shandong (2011YD19005; 2011YD21022) and Natural Scientific Foundation of Chinese Shandong Province (ZR2010CQ031).

\section{References}

1. Messina M: A brief historical overview of the past two decades of soy and isoflavone research. J Nutr 140: S1350-S1354, 2010.

2. Kao TH and Chen BH: Functional components in soybean cake and their effects on antioxidant activity. J Agric Food Chem 54: 7544-7555, 2006.

3. Kao TH, Huang RFS and Chen BH: Antiproliferation of hepatoma cell and progression of cell cycle as affected by isoflavone extracts from soybean cake. Int J Mol Sci 8: 1095-1110, 2007.

4. Kao TH, Wu WM, Hung CF, Wu WB and Chen BH: Anti- inflammatory effects of isoflavone powder produced from soybean cake. J Agric Food Chem 55: 11068-11079, 2007.

5. Dalais FS, Ebeling PR, Kotsopoulos D, McGrath BP and Teede HJ: The effects of soy protein containing isoflavones on lipids and indices of bone resorption in postmenopausal women. Clin Endocrinol (Oxf) 58: 704-709, 2003.

6. Migliaccio S and Anderson JJ: Isoflavones and skeletal health: are these molecules ready for clinical application? Osteoporos Int 14: 361-368, 2003.

7. Farina HG, Pomies M, Alonso DF and Gomez DE: Antitumor and antiangiogenic activity of soy isoflavone genistein in mouse models of melanoma and breast cancer. Oncol Rep 16: 885-891, 2006.

8. Wada K, Nakamura K, Tamai Y, et al: Soy isoflavone intake and breast cancer risk in Japan: From the Takayama study. Int J Cancer: Feb 6, 2013 (Epub ahead of print). doi: 10.1002/ijc.28088

9. Boucher BA, Cotterchio M, Anderson LN, Kreiger N, Kirsh VA and Thompson LU: Use of isoflavone supplements is associated with reduced postmenopausal breast cancer risk. Int J Cancer 132: 1439-1450, 2013.
10. Zhang YF, Kang HB, Li BL and Zhang RM: Positive effects of soy isoflavone food on survival of breast cancer patients in China. Asian Pac J Cancer Prev 13: 479-482, 2012.

11. Satoh H, Nishikawa K, Suzuki K, et al: Genistein, a soy isoflavone, enhances necrotic-like cell death in a breast cancer cell treated with a chemotherapeutic agent. Res Commun Mol Pathol Pharmacol 113-114: 149-158, 2003.

12. Xie Q, Chen ML, Qin Y, et al: Isoflavone consumption and risk of breast cancer: a dose-response meta-analysis of observational studies. Asia Pac J Clin Nutr 22: 118-127, 2013.

13. Akaza H, Miyanaga N, Takashima N, et al: Is daidzein non-metabolizer a high risk for prostate cancer? A case-controlled study of serum soybean isoflavone concentration. Jpn J Clin Oncol 32: 296-300, 2002.

14. Kurahashi N, Iwasaki M, Sasazuki S, Otani T, Inoue M and Tsugane S: Soy product and isoflavone consumption in relation to prostate cancer in Japanese men. Cancer Epidemiol Biomarkers Prev 16: 538-545, 2007.

15. Perabo FG, Von Low EC, Ellinger J, von Rucker A, Muller SC and Bastian PJ: Soy isoflavone genistein in prevention and treatment of prostate cancer. Prostate Cancer Prostatic Dis 11: 6-12, 2008.

16. Sugiyama Y, Masumori N, Fukuta F, et al: Influence of isoflavone intake and equol-producing intestinal flora on prostate cancer risk. Asian Pac J Cancer Prev 14: 1-4, 2013.

17. File SE, Jarrett N, Fluck E, Duffy R, Casey K and Wiseman H: Eating soya improves human memory. Psychopharmacology (Berl) 157: 430-436, 2001.

18. Bone HG, Greenspan SL, McKeever C, et al: Alendronate and estrogen effects in postmenopausal women with low bone mineral density. Alendronate/Estrogen Study Group. J Clin Endocrinol Metab 85: 720-726, 2000.

19. Gallagher JC: The pathogenesis of osteoporosis. Bone Miner 9: 215-227, 1990.

20. Wei P, Liu M, Chen Y and Chen DC: Systematic review of soy isoflavone supplements on osteoporosis in women. Asian Pac J Trop Med 5: 243-248, 2012.

21. Verhaeghe J, van Bree R, Van Herck E, et al: Effects of recombinant human growth hormone and insulin-like growth factor-I, with or without 17 beta-estradiol, on bone and mineral homeostasis of aged ovariectomized rats. J Bone Miner Res 11: 1723-1735, 1996.

22. Nielsen TF, Ravn P, Bagger YZ, Warming L and Christiansen C: Pulsed estrogen therapy in prevention of postmenopausal osteoporosis. A 2-year randomized, double blind, placebo-controlled study. Osteoporos Int 15: 168-174, 2004.

23. Seeman E: Estrogen, androgen, and the pathogenesis of bone fragility in women and men. Curr Osteoporos Rep 2: 90-96, 2004.

24. Celec P, Ostatnikova D, Caganova M, et al: Endocrine and cognitive effects of short-time soybean consumption in women. Gynecol Obstet Invest 59: 62-66, 2005.

25. Kritz-Silverstein D, Von Muhlen D, Barrett-Connor E and Bressel MA: Isoflavones and cognitive function in older women: the SOy and Postmenopausal Health In Aging (SOPHIA) Study. Menopause 10: 196-202, 2003.

26. Lephart ED, West TW, Weber KS, et al: Neurobehavioral effects of dietary soy phytoestrogens. Neurotoxicol Teratol 24: 5-16, 2002.

27. Lund TD, West TW, Tian LY, et al: Visual spatial memory is enhanced in female rats (but inhibited in males) by dietary soy phytoestrogens. BMC Neurosci 2: 20, 2001.

28. Lee YB, Lee HJ, Won MH, et al: Soy isoflavones improve spatial delayed matching-to-place performance and reduce cholinergic neuron loss in elderly male rats. J Nutr 134: 1827-1831, 2004.

29. Bang OY, Hong HS, Kim DH, et al: Neuroprotective effect of genistein against beta amyloid-induced neurotoxicity. Neurobiol Dis 16: 21-28, 2004.

30. Behl C: Alzheimer's disease and oxidative stress: implications for novel therapeutic approaches. Prog Neurobiol 57: 301-323, 1999.

31. Holscher C: Possible causes of Alzheimer's disease: amyloid fragments, free radicals, and calcium homeostasis. Neurobiol Dis 5: 129-141, 1998.

32. Morris JC: Dementia update 2003. Alzheimer Dis Assoc Disord 17: 245-258, 2003.

33. Ramassamy C, Averill D, Beffert U, et al: Oxidative insults are associated with apolipoprotein E genotype in Alzheimer's disease brain. Neurobiol Dis 7: 23-37, 2000.

34. Zeng H, Chen Q and Zhao B: Genistein ameliorates beta-amyloid peptide (25-35)-induced hippocampal neuronal apoptosis. Free Radic Biol Med 36: 180-188, 2004. 
35. Pan Y, Anthony M and Clarkson TB: Effect of estradiol and soy phytoestrogens on choline acetyltransferase and nerve growth factor mRNAs in the frontal cortex and hippocampus of female rats. Proc Soc Exp Biol Med 221: 118-125, 1999.

36. Xi YD, Li XY, Ding J, et al: Soy isoflavone alleviates A $31-42$-induced impairment of learning and memory ability through the regulation of RAGE/LRP-1 in neuronal and vascular tissue. Curr Neurovasc Res 10: 144-156, 2013.

37. Tikkanen MJ and Adlercreutz H: Dietary soy-derived isoflavone phytoestrogens. Could they have a role in coronary heart disease prevention? Biochem Pharmacol 60: 1-5, 2000.

38. Beavers DP, Beavers KM, Miller M, Stamey J and Messina MJ Exposure to isoflavone-containing soy products and endothelial function: a Bayesian meta-analysis of randomized controlled trials. Nutr Metab Cardiovasc Dis 22: 182-191, 2012.

39. Yamakoshi J,Piskula MK, Izumi T, et al: Isoflavone aglycone-rich extract without soy protein attenuates atherosclerosis development in cholesterol-fed rabbits. J Nutr 130: 1887-1893, 2000

40. Chan YH, Lau KK, Yiu KH, et al: Reduction of C-reactive protein with isoflavone supplement reverses endothelial dysfunction in patients with ischaemic stroke. Eur Heart J 29: 2800-2807, 2008.

41. Gilbert ER and Liu D: Anti-diabetic functions of soy isoflavone genistein: mechanisms underlying its effects on pancreatic $\beta$-cell function. Food Funct 4: 200-212, 2013.

42. Behloul $\mathrm{N}$ and $\mathrm{Wu}$ G: Genistein: a promising therapeutic agent for obesity and diabetes treatment. Eur J Pharmacol 698: 31-38, 2013.

43. Valsecchi AE, Franchi S, Panerai AE, Rossi A, Sacerdote P and Colleoni M: The soy isoflavone genistein reverses oxidative and inflammatory state, neuropathic pain, neurotrophic and vasculature deficits in diabetes mouse model. Eur J Pharmacol 650: 694-702, 2011.
44. Chiang HS, Wu WB, Fang JY, et al: UVB-protective effects of isoflavone extracts from soybean cake in human keratinocytes. Int J Mol Sci 8: 651-661, 2007.

45. Chiu TM, Huang CC, Lin TJ, Fang JY, Wu NL and Hung CF In vitro and in vivo anti-photoaging effects of an isoflavone extract from soybean cake. J Ethnopharmacol 126: 108-113, 2009.

46. Assefa Z, Van Laethem A, Garmyn M and Agostinis P: Ultraviolet radiation-induced apoptosis in keratinocytes: on the role of cytosolic factors. Biochim Biophys Acta 1755: 90-106, 2005.

47. Portman MA: Kawasaki disease and soy: potential role for is oflavone interaction with $\mathrm{Fcg} \gamma$ receptors. Pediatr Res 73: 130-134, 2013.

48. Safe S: Endocrine disruptors and human health: is there a problem. Toxicology 205: 3-10, 2004.

49. Paganetto G, Campi F, Varani K, Piffanelli A, Giovannini G and Borea PA: Endocrine-disrupting agents on healthy human tissues. Pharmacol Toxicol 86: 24-29, 2000.

50. Wuttke W, Jarry H, Becker T, et al: Phytoestrogens: endocrine disrupters or replacement for hormone replacement therapy? Maturitas 44 (Suppl 1): S9-S20, 2003.

51. Helferich WG, Andrade JE and Hoagland MS: Phytoestrogens and breast cancer: a complex story. Inflammopharmacology 16: 219-226, 2008

52. Chen A and Rogan WJ: Isoflavones in soy infant formula: a review of evidence for endocrine and other activity in infants. Annu Rev Nutr 24: 33-54, 2004. 